Диагностика отрыва клапаносодержащего кондуита восходящего отдела аорты и его успешное хирургическое лечение

\author{
Малахова М.В., Прохорова Е.А., Куличкин А.С., Бриль К.Р., Ван Е.Ю., \\ Дзеранова А.Н., , Галян Т.Н., Ховрин В.В., Чарчян Э.Р.
}

ФГБНУ «Российский научный центр хирургии им. акад. Б.В. Петровского», Абрикосовский пер., 2, Москва, 119991, Российская Федерация

\begin{abstract}
Малахова Мария Владимировна, врач-рентгенолог ФГБНУ «Российский научный центр хирургии им. акад. Б.В. Петровского»; http://orcid.org/0000-0002-9985-3753
\end{abstract}

Прохорова Екатерина Алексеевна, К. М. Н., врач-рентгенолог ФГБНУ «Российский научный центр хирургии им. акад. Б.В. Петровского»; http://orcid.org/0000-0001-9788-5666

Куличкин Алексей Сергеевич, врач-рентгенолог ФГБНУ «Российский научный центр хирургии им. акад. Б.В. Петровского»; http://orcid.org/0000-0003-1256-560X

Бриль Кристина Руслановна, аспирант ФГБНУ «Российский научный центр хирургии им. акад. Б.В. Петровского»; http://orcid.org/0000-0001-8789-0330

Ван Елена Юрьевна, к. М .н., врач ультразвуковой диагностики ФГБНУ «Российский научный центр хирургии им. акад. Б.В. Петровского»;

http://orcid.org/0000-0002-4399-0716

Дзеранова Ангелина Николаевна, к. М. Н., науч. сотр. ФГБНУ «Российский научный центр хирургии им. акад. Б.В. Петровского»; http://orcid.org/0000-0002-7916-1151

Галян Татьяна Николаевна, к. М. Н., заведующая отделением рентгенодиагностики и компьютерной томографии ФГБНУ «Российский научный центр хирургии им. акад. Б.В. Петровского»; http://orcid.org/0000-0003-4751-5119

Ховрин Валерий Владиславович, д. М. Н., гл. науч. сотр. ФГБНУ «Российский научный центр хирургии им. акад. Б.В. Петровского»; http://orcid.org/0000-0002-6720-4126

Чарчян Эдуард Рафаэлович, д. М. Н., заведующий 1-м кардиохирургическим отделением ФГБНУ «Российский научный центр хирургии им. акад. Б.В. Петровского»;

http://orcid.org/0000-0003-3164-2877

\begin{abstract}
Резюме
Инфицирование протеза является наиболее частым осложнением после протезирования аорты синтетическим сосудистым протезом или клапаносодержащим кондуитом (КСК), при этом отрыв протеза аортального клапана (АК) встречается в 0,1-1,3\% случаев. Причиной отрыва клапана является не только инфекционный эндокардит. Аневризма восходящей аорты и выраженная кальцификация нативного АК также относятся к известным факторам риска. В статье представлен клинический случай обследования и лечения пациента 49 лет в условиях РНЦХ имени акад. Б.В. Петровского. Через 18 лет после первичного оперативного вмешательства по поводу аортального стеноза было проведено репротезирование АК и протезирование восходящей аорты КСК по методике Бенталла - Де Боно в модификации Кучукоса. В 2021 г. по данным компьютерной томографической аортографии на фоне инфекционного эндокардита выявлен отрыв протеза аорты с формированием парапротезной фистулы и полости ложной аневризмы. Результаты инструментальных методов исследований сопоставимы с интраоперационными данными. Клинический случай представляет интерес ввиду редкой встречаемости осложнения и значимой роли мультиспиральной компьютерной томографии при обследовании и послеоперационном контроле.

Ключевые слова: компьютерная томография; компьютерная томографическая аортография; протезирование аортального клапана; инфицирование протеза; инфекционный эндокардит.

Конфликт интересов. Авторы заявляют об отсутствии конфликта интересов.

Участие авторов. Авторы внесли равноценный вклад в подготовку статьи для публикации.

Для цитирования: Малахова М.В., Прохорова Е.А., Куличкин А.С., Бриль К.Р., Ван Е.Ю., Дзеранова А.Н., Галян Т.Н., Ховрин В.В., Чарчян Э.Р. Диагностика отрыва клапаносодержащего кондуита восходящего отдела аорты и его успешное хирургическое лечение. Вестник рентгенологии и радиологии. 2021; 102(6): 383-9. https://doi.org/10.20862/0042-4676-2021-102-6-383-389

Для корреспонденции: Малахова Мария Владимировна, E-mail:mmv989@yandex.ru
\end{abstract}




\title{
Diagnosis and Successful Surgical Treatment of Ascending Aorta Valved Conduit Rupture
}

\author{
Maria V. Malakhova, Ekaterina A. Prokhorova, Aleksey S. Kulichkin, Kristina R. Bril', \\ Elena Yu. Van, Angelina N. Dzeranova, Tatiana N. Galyan, Valeriy V. Khovrin, \\ Eduard R. Charchyan
}

Petrovsky Russian Scientific Center of Surgery,

Abrikosovskiy per., 2, Moscow, 119991, Russian Federation

\begin{abstract}
Maria V. Malakhova, Radiologist, Petrovsky Russian Scientific Center of Surgery; http://orcid.org/0000-0002-9985-3753
\end{abstract}

Ekaterina A. Prokhorova, Cand. Med. Sc., Radiologist, Petrovsky Russian Scientific Center of Surgery; http://orcid.org/0000-0001-9788-5666

Aleksey S. Kulichkin, Radiologist, Petrovsky Russian Scientific Center of Surgery; http://orcid.org/0000-0003-1256-560X

Kristina R. Bril', Postgraduate, Petrovsky Russian Scientific Center of Surgery; http://orcid.org/0000-0001-8789-0330

Elena Yu. Van, Cand. Med. Sc., Ultrasound Diagnostician, Petrovsky Russian Scientific Center of Surgery; http://orcid.org/0000-0002-4399-0716

Angelina N. Dzeranova, Cand. Med. Sc., Researcher, Petrovsky Russian Scientific Center of Surgery; http://orcid.org/0000-0002-7916-1151

Tatiana N. Galyan, Cand. Med. Sc., Head of Department of Radiology and Computed Tomography, Petrovsky Russian Scientific Center of Surgery; http://orcid.org/0000-0003-4751-5119

Valeriy V. Khovrin, Dr. Med. Sc., Senior Researcher, Petrovsky Russian Scientific Center of Surgery; http://orcid.org/0000-0002-6720-4126

Eduard R. Charchyan, Dr. Med. Sc., Head of Cardiac Surgery Department No. 1, Petrovsky Russian Scientific Center of Surgery; http://orcid.org/0000-0003-3164-2877

\begin{abstract}
Prosthetic infection is the most common complication after aortic replacement with a synthetic vascular prosthesis or a valved conduit (VC); in this case, aortic valve (AV) rupture occurs in $0.1-1.3 \%$ of patients. The cause of valve rupture is not only infective endocarditis; ascending aortic aneurysm and obvious calcification of the native AV are also known risk factors. The paper describes a clinical case of a 49-yearold patient examined and treated in Petrovsky Russian Scientific Center of Surgery. Eighteen years after primary surgical intervention for aortic stenosis, $\mathrm{AV}$ reprosthesis and ascending aorta $\mathrm{VC}$ replacement were performed according to the Bentall - De Bono procedure modified by Kouchoukos. In 2021, computed tomographic aortography has revealed that in the presence of infective endocarditis, there is aortic prosthetic rupture to form a paraprosthetic fistula and a pseudoaneurysm cavity. The results of instrumental studies are comparable with intraoperative data. The clinical case is of interest due to the rare occurrence of complications and to the significant role of multislice computed tomography during examination and postoperative monitoring.

Keywords: computed tomography; computed tomographic aortography; aorta valve replacement; graft infection; infectious endocarditis.

Conflict of interest. The authors declare no conflict of interest.

Author contributions. The authors made an equal contribution to the preparation of the article for publication. For citation: Malakhova MV, Prokhorova EA, Kulichkin AS, Bril' KR, Van EYu, Dzeranova AN, Galyan TN, Khovrin VV, Charchyan ER. Diagnosis and successful surgical treatment of ascending aorta valved conduit rupture. Journal of Radiology and Nuclear Medicine. 2021; 102(6): 383-9 (in Russian). https://doi.org/10.20862/0042-4676-2021-102-6-383-389
\end{abstract}

For corresponding: Mariya V. Malakhova, E-mail:mmv989@yandex.ru 


\section{Введение}

Количество повторных операций на аортальном клапане (АК) и восходящей аорте (ВАо) возросло. До 10\% больных, которым ранее было выполнено протезирование ВАо и АК, подвергаются повторной операции на корне аорты в ближайшем и отдаленном периодах [1-3].

Отрыв протеза АК представляет собой редкое осложнение, возникающее в 0,1-1,3\% случаев. Известными факторами риска отрыва клапана являются инфекционный эндокардит, сопутствующая аневризма ВАо и выраженная кальцификация нативного АК [4]. Описаны редкие случаи отрыва клапаносодержащего кондуита (КСК) ВАо на фоне позднего инфекционного эндокардита после операции Бенталла - Де Боно [5, 6].

Мультиспиральная компьютерная томография (МСКТ) аорты с внутривенным контрастированием (КТ-аортография) является признанным стандартом для диагностики первичной патологии аорты и осложнений после реконструктивных операций на аорте [7].

\section{Описание случая}

ПациентШ. 49 лет поступил в РНЦХ им. акад. Б.В. Петровского в экстренном порядке с жалобами на выраженную одышку при незначительных физических нагрузках, сухой кашель. Ухуд-

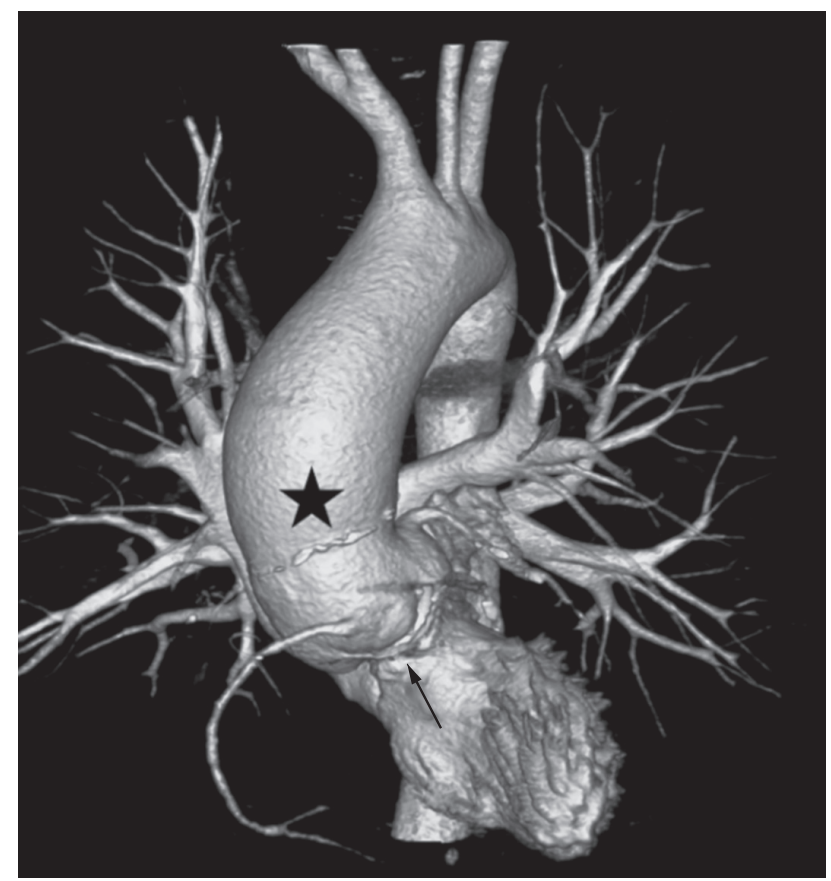

Puc. 1. КТ-аортография, 3D-реконструкция. Состояние после протезирования аортального клапана. Стрелкой указан протез аортального клапана, звездочкой - аневризма восходящей аорты

Fig. 1. CT aortography, 3D reconstruction. Status after aortic valve replacement. The arrow indicates aortic valve prosthesis; the asterisk shows ascending aortic aneurysm шение состояния отмечалось в течение последних 6 мес, когда прогрессивно снижалась толерантность к физическим нагрузкам с приступами ортопноэ, нарастали симптомы астении, появились отеки нижних конечностей, снижение артериального давления до 110-100/50-40 мм рт. ст.

Из анамнеза: в 2000 г. - протезирование АK механическим протезом Carbonyx по поводу аортального стеноза. В 2018 г. выполнена КТ-аортография и выявлена аневризма ВАо (рис. 1). В том же году проведено репротезирование АК и протезирование ВАо КСК по методике Бенталла Де Боно в модификации Кучукоса. Послеоперационный период протекал без осложнений.

При поступлении в РНЦХ им. акад. Б.В. Петровского пациент был обследован.

трансторакальная эхокардиография (ЭхоКГ). В позиции ВАо визуализируется КСК, диаметр протеза 33 мм, равномерный при цветовом допплеровском картировании (ЦДК) на всем протяжении. В проекции митрально-аортального контакта визуализируется парапротезная фистула (ППФ). Эксцентричная парапротезная струя регургитации в проекции митрально-аортального контакта (2-3-й ст., оценка затруднена). Сообщение между полостью левого желудочка и парапротезным пространством (ППП) ВАо, ширина по задней стенке 17 мм, ширина по передней стенке 11 мм. Стенки

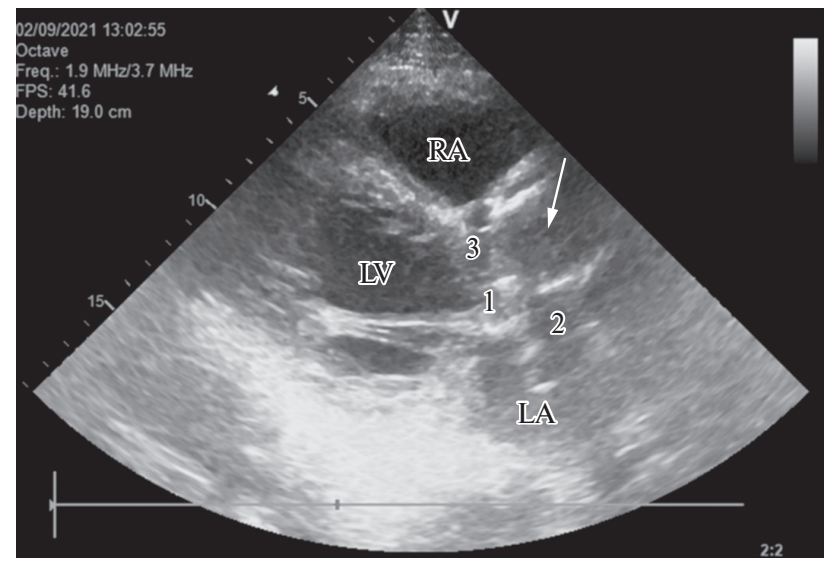

Puc. 2. Трансторакальная эхокардиограмма до операции (парастернальная позиция по длинной оси сердца). Стрелкой указан клапаносодержащий кондуит восходящей аорты. RA - правый желудочек; LV - левый желудочек; LA - левое предсердие; 1 - парапротезная фистула в области митрально-аортального контакта; 2 - псевдоаневризма; 3 - парапротезная фистула по передней стенке аорты

Fig. 2. Transthoracic echocardiogram before surgery (the parasternal position along the long axis of the heart). The arrow indicates an ascending aortic valved conduit.

RA - right ventricle; LV - left ventricle; LA - left atrium; 1 - paraprosthetic fistula in the mitral-aortic contact area; 2 - pseudoaneurysm; 3 - paraprosthetic fistula along the anterior aortic wall 

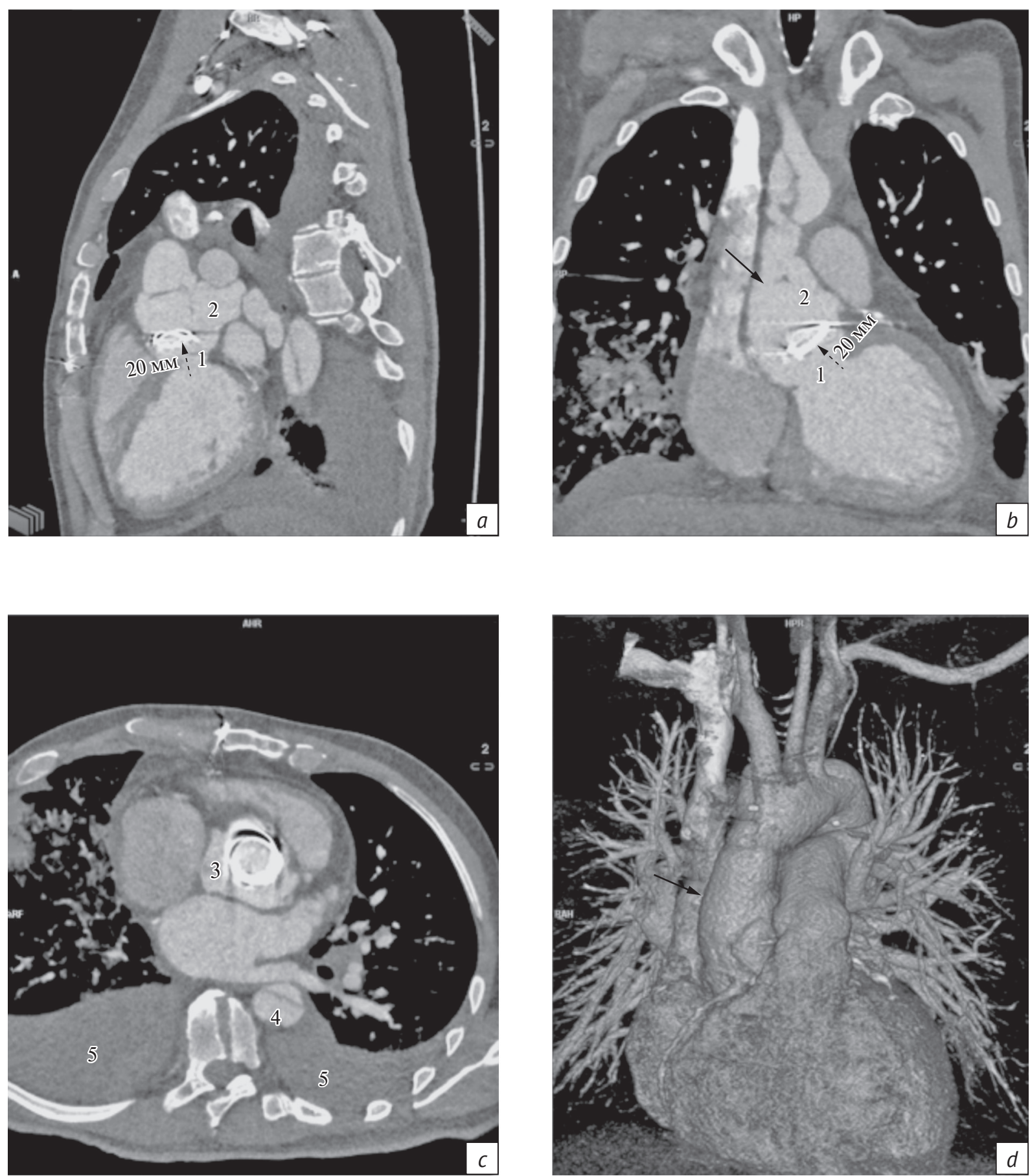

Puс. 3. КТ-аортография с ЭхоКГ-синхронизацией. Область исследования 661 мм, лучевая нагрузка 18,4 м3в, объем контрастного вещества 98 мл: $a$ - сагиттальная проекция; $b$ - коронарная проекция, $c$ - аксиальная проекция на уровне локализации механического протеза клапаносодержащего кондуита; $d$ - 3D-реконструкция.

1 - фиброзное кольцо аортального клапана; 2 - полость ложной аневризмы аорты; 3 - расширенное и контрастное парапротезное пространство; 4 - расслоение нисходящей аорты; 5 - двусторонний гидроторакс.

Сплошными стрелками указан клапаносодержащий кондуит восходящей аорты, пунктирными - дислокация на 20 мм его механического протеза

Fig. 3. CT aortography with EchoCG synchronization. A study area is $661 \mathrm{~mm}$; a radiation exposure is $18.4 \mathrm{~m} 3 \mathrm{v}$; a contrast medium volume is $98 \mathrm{ml}$ :

$a$ - sagittal projection; $b$ - coronal projection; $c$ - axial projection at the level of localization of a mechanical prosthetic valved conduit; $d-3 \mathrm{D}$ reconstruction.

1 - aortic valve fibrous ring; 2 - aortic pseudoaneurysm cavity; 3 - extended and contrast paraprosthetic space; 4 - descending aortic dissection; 5 - bilateral hydrothorax.

The solid arrows indicate the ascending aorta valved conduit; the dotted arrows show the 20-mm dislocation of its mechanical prosthesis 


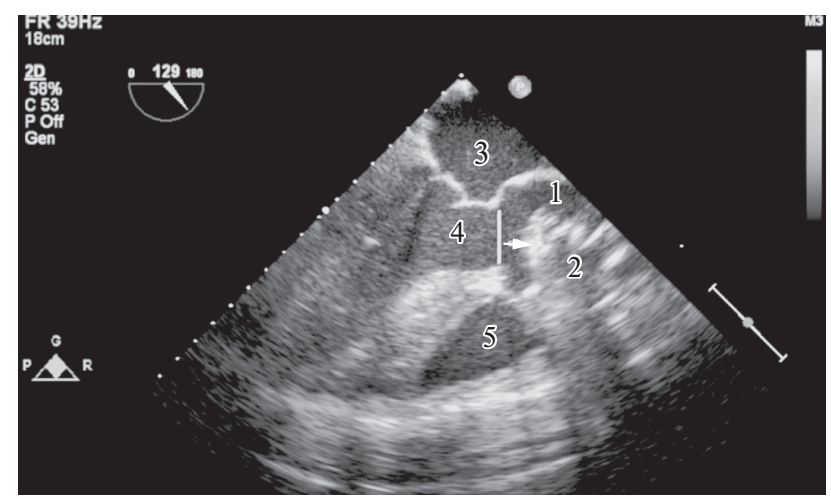

Рис. 4. Чреспищеводная ЭхоКГ, В-режим.

1 - парапротезное пространство восходящей аорты; 2 просвет протеза восходящей аорты; 3 - левое предсердие; 4 - выводной тракт левого желудочка; 5 - правый желудочек. Вертикальной линией обозначена проекция фиброзного кольца аортального клапана, стрелкой - механический протез клапаносодержащего кондуита восходящей аорты

Fig. 4. Transesophageal echocardiography in B-mode.

1 - paraprosthetic space of the ascending aorta; 2 - the lumen of the ascending aortic prosthesis; 3 - left atrium; 4 - left ventricular outflow tract; 5 - right ventricle.

The vertical line indicates the projection of aortic valve fibrous ring; the arrow shows the mechanical prosthesis of an ascending aorta valved conduit

протеза ВАо в систолу гипермобильны. Дистальный анастомоз не визуализируется. Заключение: ППФ. Аортальная недостаточность 2-3-й ст. Митральная недостаточность 2-3-й ст. Снижение глобальной и локальной систолических функций гипертрофированного левого желудочка. Выраженная дилатация левого желудочка, дилатация левого предсердия. Трикуспидальная недостаточность 1-2-й ст. Двусторонний гидроторакс, выраженный справа (рис. 2).

КT-аортография. Дислокация протеза АК на 20 мм дистальнее фиброзного кольца АК, контрастирование циркулярно расширенного до 22 мм ППП (общий диаметр аорты $68 \times 44$ мм), по задней стенке сформирована полость ложной аневризмы $23 \times 44$ мм. Коронарные артерии проливаются от протеза. Верхняя граница ложной аневризмы на уровне 63 мм от фиброзного кольца. Формирование «двойного канала» в аорте от уровня дистального анастомоза протеза с распространением на устье брахиоцефального ствола и на всем протяжении дуги и нисходящей торакоабдоминальной аорты с дистальной фенестрацией в инфраренальной аорте. Дуга аорты и брюшная аорта не расширены. Отмечается диссекция в устье правой почечной артерии. Мальперфузии почек не выявлено. В терминальной аорте и общих подвздошных артериях «двойного канала» не выявлено. Заключение: отрыв протеза аорты с дислокацией, формированием ППФ и полости ложной аневризмы на фоне инфекционного эндокардита. Расслоение дуги и нисходящей аорты (НАо) с распространением на устье брахиоцефального ствола и устье правой почечной артерии. Двусторонняя нижнедолевая пневмония, двусторонний гидроторакс (рис. 3).

Интраоперационная ЭхоКГ. Протез ВАо находится в ППП, полуконцентрически не определяется фиксация протеза, работа запирательного механизма не нарушена. В области митрально-аортального контакта при ЦДК определяется фистула в ППП по задней стенке протеза аорты. Устья коронарных сосудов визуализированы. При ЦДК: трикуспидальная недостаточность 2-3-й ст. (рис. 4).

Экстренная операция. Выполнено периферическое подключение искусственного кровообращения через бедренную вену и подключичную артерию, повторная продольная срединная стернотомия. Отмечен выраженный спаечный процесс в средостении, дифференцировка тканей нарушена. С техническими трудностями мобилизованы протез ВАо, дуга аорты, устья брахиоцефальных ветвей, поперечная вена, взяты на тесьму. После удаления всех инфицированных тканей и синтетических протезов выполнены протезирование митрального клапана, ушивание открытого овального окна и пластика трикуспидального клапана по Батисте (рис. 5).

В фиброзное кольцо АК имплантирован аортальный гомографт (АГФ). Устья коронарных артерий реимплантированы в бок АГФ. На фоне циркуляторного ареста и антеградной перфузии головного мозга в истинный канал НАо низведен протез Polythese c рентгеноконтрастными метками и выполнено протезирование дуги с ветвями единым гомографтом. Гладкий послеоперационный период.

Контрольная КТ-аортография. Контрастный просвет АГФ и дуга аорты без дефектов контрастирования. Эндоликинга контрастного вещества не выявлено. Дистальное расслоение НАо от уровня дистального анастомоза протеза с фенестрацией

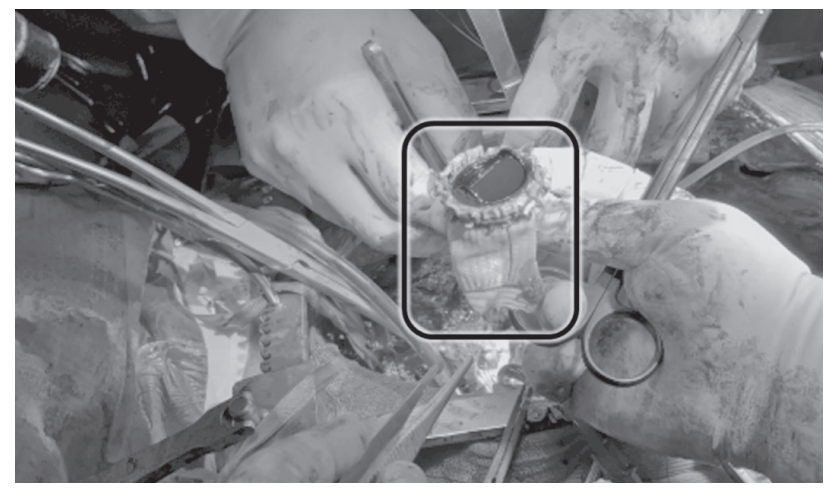

Puc. 5. Интраоперационное фото на этапе удаления клапаносодержащего кондуита восходящей аорты

Fig. 5. Intraoperative photo at the stage of ascending aorta valved conduit removal 


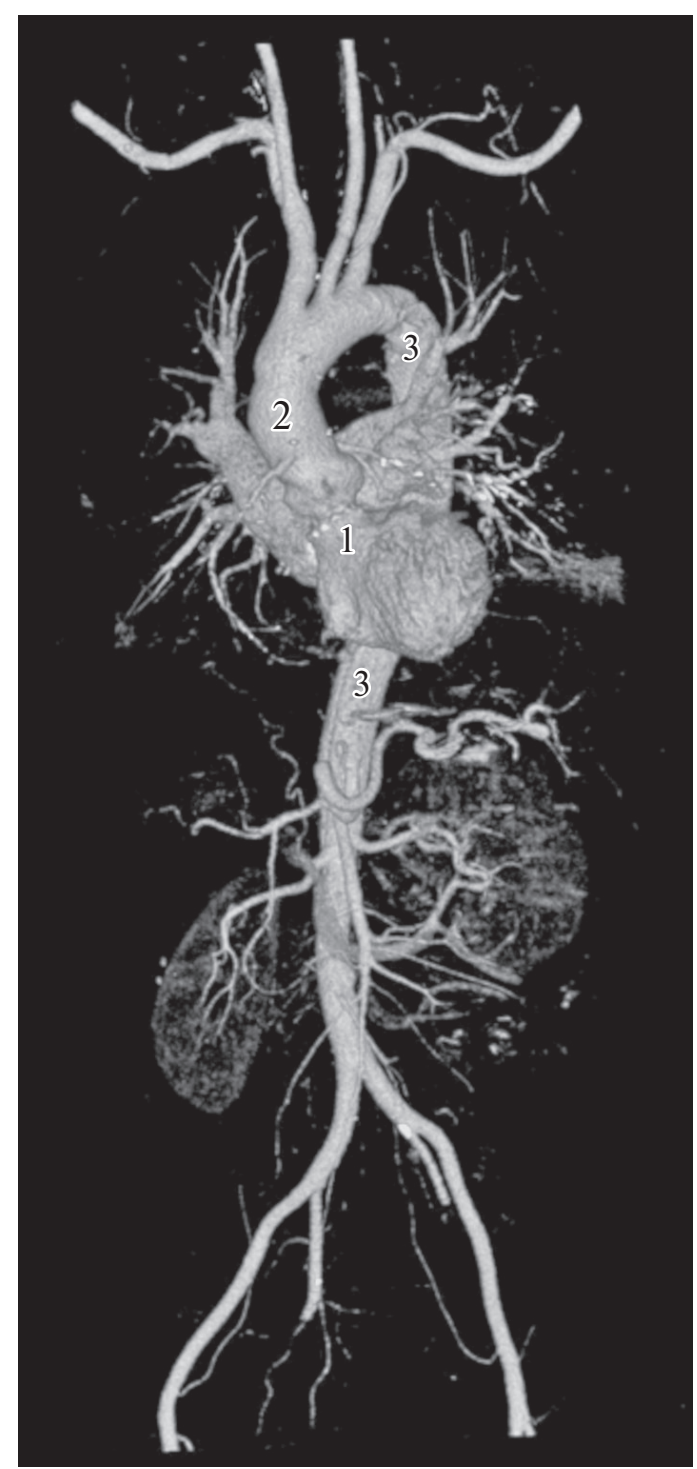

Puc. 6. КТ-аортография (послеоперационный контроль) без электрокардиографической синхронизации, 3D-реконструкция. Область исследования 664 мм, лучевая нагрузка 5,9 м3в, объем контрастного вещества 60 мл.

1 - фиброзное кольцо аортального клапана; 2 - аортальный гомографт; 3 - расслоение нисходящей аорты

Fig. 6. СТ aortography (postoperative control) without ECG synchronization, 3D reconstruction. A study area is $664 \mathrm{~mm}$; a radiation exposure is $5.9 \mathrm{m3v}$; a contrast medium volume is $60 \mathrm{ml}$.

1 - aortic valve fibrous ring; 2 - aortic homograft; 3 descending aorta dissection

в инфраренальном отделе брюшной аорты. Торакоабдоминальная аорта не расширена (рис. 6).

Контрольная трансторакальная ЭхоКГ с ЦДК. В позиции ВАо визуализируется АГФ на расстоянии, диаметр просвета гомографта 32 мм, равномерно прокрашивается при ЦДК, по передней стенке гомографта визуализируется ППП шириной 8 мм. При ЦДК регургитации нет. В позиции митрального клапана механический протез.

\section{Обсуждение}

Среди всех повторных вмешательств на ВАо и АК частота операций у пациентов с инфекционными осложнениями по данным разных авторов составляет от $21 \%$ до $61 \%$. Проведение детального анализа состояния КСК ВАо, оценка объема предстоящего оперативного вмешательства на фоне сопутствующей тяжести состояния пациента и вынужденной ургентности поставленной задачи требуют высокой точности и скорости проводимого обследования. Техническое выполнение трансторакальной ЭхоКГ и МСКТ-аортографии не представляет серьезных трудностей, однако анализ состояния кондуита на фоне турбулентных разнонаправленных потоков в данной ситуации - непростая задача для ультразвуковых методов исследования, требующая более тщательного анализа и времени. Поэтому проведение КТ-аортографии c электрокардиографической синхронизацией и последующим 3D-моделированием на дооперационном этапе оправдано меньшим временем для проведения и анализа данных МСКТ. При этом метод обладает наивысшей точностью и высоко информативен не только для характеристики объема поражения в зоне инфекционного процесса, но и для уточнения протяженности и распространения патологических изменений на всем протяжении аорты.

Для необходимого анализа и определения тактики ведения пациента из объема подробной информации при КТ-аортографии важно: отметить расположение КСК ВАо по отношению к позиции фиброзного кольца АК; оценить протяженность деструкции тканей при инфекционном эндокардите с объемом ППФ, размерами ППП и полости ложных аневризм; охарактеризовать проходимость брахиоцефальных ветвей аорты и состояние ранее сформированных анастомозов и сегментов торакоабдоминальной аорты, выявляя наличие признаков мальперфузии органов. Выбор тактики, метода и объема хирургического вмешательства осуществляется исходя из полученных диагностических данных и клинического состояния пациента. Интраоперационный контроль выполняется посредством чреспищеводной ЭхоКГ.

\section{Заключение}

Требования к объему обследования пациентов с инфекционным эндокардитом после реконструктивных операций на аорте выделяют особое место и важность КТ-аортографии, являющейся ключевым звеном в коррекции диагноза, выборе объема повторного хирургического вмешательства и послеоперационном контроле. 


\section{Литература}

1. Белов Ю.В., Чарчян Э.Р., Степаненко А.Б. Повторные операции после протезирования аортального клапана и восходящей аорты. Кардиология и сердечно-сосудистая хирургия. 2008; 1(2): 37-40.

2. Малашенков А.И., Русанов Н.И., Кокоев М.Б. и др. Анализ повторных операций на восходящей аорте после ранее выполненных вмешательств на восходящей аорте и аортальном клапане. Анналы хирургии. 2010; 3: 40-3.

3. Скопин И.И., Кахкцян П.В., Алексанян Г.Г. Повторная операция Бенталл-Де Боно после ранее выполненного протезирования восходящей аорты и аортального клапана. Кардиология и сердечно-сосудистая хирургия. 2017; 10(5): 78-80. https://doi.org/10.17116/kardio201710578-80.

4. Mancio J, Almeida C, Pires-Morais G, et al. Hanging by a thread: major detachment of an aortic prosthetic valve. Rev Port
Cardiol. 2015; 34(12): 787-8.

http://dx.doi.org/10.1016/j.repc.2015.07.009.

5. Сорока Н.В., Болдырев С.Ю., Лайпанова Л.К., Шелестова И.А. Случай позднего вторичного инфекционного эндокардита у пациента после операции Бенталла Де Боно. Инновационная медицина Кубани. 2016; 3: 37-41.

6. Морова Н.А., Цеханович В.Н. Особенности течения инфекционного эндокардита после протезирования восходящей аорты клапаносодержащим кондуитом. Архивъ внутренней медицины. 2019; 9(6): 450-9. https://doi.org/10.20514/2226-6704-2019-9-6-450-459.

7. Ховрин В.В., Галян Т.Н., Малахова М.В. и др. «Сверхбыстрая» МСКТ-аортография: реальность и перспектива. Медицинская визуализация. 2017; 21(4): 33-40. https://doi.org/10.24835/1607-0763-2017-4-33-40.

\section{References}

1. Belov luV, Charchian ER, Stepanenko AB. Repeated operations after aortic valve and ascending aorta replacement. Russian Journal of Cardiology and Cardiovascular Surgery. 2008; 1(2): 37-40 (in Russ.).

2. Malashenkov Al, Rusanov NI, Kokoev MB, et al. Analysis of repeated operations on the ascending aorta after previously performed interventions on the ascending aorta and aortic valve. Russian Journal of Surgery. 2010; 3: 40-3 (in Russ.).

3. Skopin II, Kakhktsyan PV, Aleksanyan GG. Redo Bentallde Bono procedure after previous ascending aortic and aortic valve replacement. Russian Journal of Cardiology and Cardiovascular Surgery. 2017; 10(5): 78-80 (in Russ.). https://doi.org/10.17116/kardio201710578-80.

4. Mancio J, Almeida C, Pires-Morais G, et al. Hanging by a thread: major detachment of an aortic prosthetic valve. Rev Port
Cardiol. 2015; 34(12): 787-8.

http://dx.doi.org/10.1016/j.repc.2015.07.009.

5. Soroka NV, Boldirev SJ, Laypanova LK, Shelestova IA. A case with late secondary infectious endocarditis in a patient after Bentall Procedure. Innovative Medicine of Kuban. 2016; 3: 37-41 (in Russ.).

6. Morova NA, Tsekhanovich VN. Infectious endocarditis course after ascending aorta replacement with valved conduit. The Russian Archives of Internal Medicine. 2019; 9(6): 450-9. https://doi.org/10.20514/2226-6704-2019-9-6-450-459.

7. Khovrin VV, Galyan TN, Malakhova MV, et al. "Ultrafast" multispiral computer tomographic angiography of aorta: current reality and perspectives. Medical Visualization. 2017; 21(4): 33-40 (in Russ.). https://doi.org/10.24835/1607-0763-2017-4-33-40. 